\title{
Technology Research on the Waterproofness and Alkali Resistance of Silane Coupling Agent Modified Fiber Glass Board
}

\author{
Guo-Zhong LU ${ }^{1, a}$, Xiu-Juan DING ${ }^{1, b,{ }^{*}}$, Xue-Song ZHENG ${ }^{1, c}$, Yue LIU ${ }^{1, d}$ and \\ Wei-Xuan ZHAO ${ }^{1, \mathrm{e}}$
}

${ }^{1}$ Beijing Building Materials Academy of Sciences Research, State Key Laboratory of Solid Waste Reuse for Building Materials, Beijing Passive Low-energy Consumption Architectural Engineering Technology Research Center, Beijing 100041, China

adingxiujuanlove@126.com

${ }^{*}$ Corresponding author

Keywords: Silane coupling agent, Fiber glass, Waterproofness, Alkali resistance.

\begin{abstract}
In this paper, the fiber glass board was treated with silicane coupling agent so that it could improve its waterproofness and alkali resistance, testing fiber glass after the infiltration treatment of silane coupling agent. And investigate the effects on key performance indicators of fiber glass by the contrast test. Test results showed that silane coupling agent could enhance the waterproofness and alkali resistance of fiber glass. It will not damage to other properties when the addition is less than 3\% and it may enhance the mechanical strength of fiber glass.
\end{abstract}

\section{Introduction}

Fiber glass is a thermal and sound insulation material that is prepared by quartz sand, dolomite, wax stone and other natural minerals as well as other chemical materials such as soda and boron through the key processes including high temperature melting, glass liquid clarification, centrifugal spinning, curing and forming. It is proud of good thermal insulation, heat insulation, sound-absorbing properties, A-grade combustion performance and light weight (easy handling, processing and installation in the construction), additionally, it has satisfactory physical and chemical properties [1-3]. As A-grade fire-proof and thermal insulation material, fiber glass is common and mature in Germany and Europe. Some domestic units have studied application of fiber glass thermal insulation technology in the exterior wall, but a lot of problems occurred in the process of using. "Short timepartial soaking water absorption" of the current fiber glass in China is more than 10-20 times of the indicator specified in the European standards and thus it can't meet thermal insulation requirements of the exterior wall. For this reason, in order to maintain thermal insulation of inorganic fiber and improve its durability, it is necessary to enhance its waterproofness. Most of methods for improving waterproofness of the inorganic fiber cotton board are to set waterproof film and waterproof layer (cloth or paper material) between the fiber layer and interface layer or spray waterproof layer between them. Although these methods have some effects, the cost is high or water-proofness is limited. Thus it is an important issue, that is, substantially enhance inorganic fiber waterproofness on the premise of slightly increasing the cost.

In addition, alkali resistance of the fiber glass board is highly concerned in the industry. As a thermal insulation material, the fiber glass is required to be coated with the mortar interface agent in the wall and most of mortar interface agents are alkali and thus alkali resistance of fiber glass will directly determine the system durability. It has an important value to study how to improve the waterproofness and alkali resistance of the inorganic fiber cotton and reduce processing cost.

In this paper, fiber glass board is treated with the silane coupling agent in a way of infiltration so as to improve waterproofness and alkali resistance of the fiber glass. Inorganic fiber cotton is a composite material. Silane coupling agent can improve the interface state between resin and glass fiber and prevent other medium from penetrating into the interface, meanwhile, a layer of coating 
layer also can be formed on the glass fiber surface. Therefore, the silane coupling agent can enhance waterproofness and alkali resistance of the fiber glass.

\section{Experiment Part}

\section{Preparation of Silane Coupling Agent}

\section{Effect of pH on Coupling of Silane Coupling Agent.}

$\mathrm{pH}$ value of silane coupling agent water solution not only can affect the final treatment result but also hydrolysis rate of silane. Silane triol, generated from silane hydrolysis, can react itself. Oligomer of double silicone and silicone generated from polycondensation does not show the coupling effect, additionally, due to its weak water soluble, it is easy to precipitate. For the relationship between polycondensation reaction rate and $\mathrm{pH}$ value, see Table 1.

Table 1. The relationship between polycondensation reaction rate and $\mathrm{pH}$

\begin{tabular}{lllll}
\hline KH550 $\left(25^{\circ} \mathrm{C}\right)$ & $\mathrm{pH}=3$ & $\mathrm{pH}=5$ & $\mathrm{pH}=7$ & $\mathrm{pH}=9$ \\
Hydrolysis time/min & 1.2 & 115 & 660 & 167 \\
\hline
\end{tabular}

It can be seen from Table 1 that in order to maintain stability of silane coupling agent, $\mathrm{pH}$ value of silane coupling agent water solution shall be limited to 7-8 range.

\section{Effect of Silane Coupling Agent concentration on Coupling.}

During the experiment process, it is found that silane coupling agent concentration also affects hydrolysis rate of its water solution. When silane coupling agent amount is more than $3 \%$, its hydrolysis rate is significantly faster and crusting phenomenon will soon appear in the solution; when the coupling agent amount is more than $5 \%$, turbidity and sedimentation occur in the solution, in this condition, it cannot be used. Therefore, concentration of silane coupling agent shall be $0.5 \%-2 \%$.

\section{Process of Using Silane Coupling Agent to Treat Fiber Glass in a Way of Infiltration}

First, prepare KH550 solution with a certain concentration and pH value and fully immerse the fiber glass in KH550 solution for $2 \mathrm{~min}$. Then dry the soaked fiber glass for $10 \mathrm{~min}$ for the purpose of removing excess infiltration agent. At last, the treated sample is placed and dried into $70^{\circ} \mathrm{C}$ oven for $20 \mathrm{~min}$.

\section{Characterization and Performance Test of the Modified Fiber Glass}

Use Japan Hitachi S-3400 electron scanning microscope to observe morphology of the fiber glass treated with silane coupling agent and investigate alkali resistance of the modified fiber glass by mass loss rate test; assess waterproofness of fiber glass by short-term water absorption changes; because some key performance indicators of the fiber glass, after infiltration, may be affected by coupling agent, adopt the contrast experiment to test the fire-proof performance of modified fiber glass, its heat conduction coefficient, vertical pullout strength and compression strength.

\section{Results and Discussion}

\section{Alkali Resistance of Silane Coupling Agent}

\section{Morphology Characterization.}

After fiber is corroded by alkali, observe the surface damage. Upon alkali solution treatment, more rough the fiber surface, the worse the alkali resistance of the fiber. Figure 1 shows scanning electron 
microscope photographs of ordinary fiber glass and those treated with silane coupling agent in a way of infiltration.
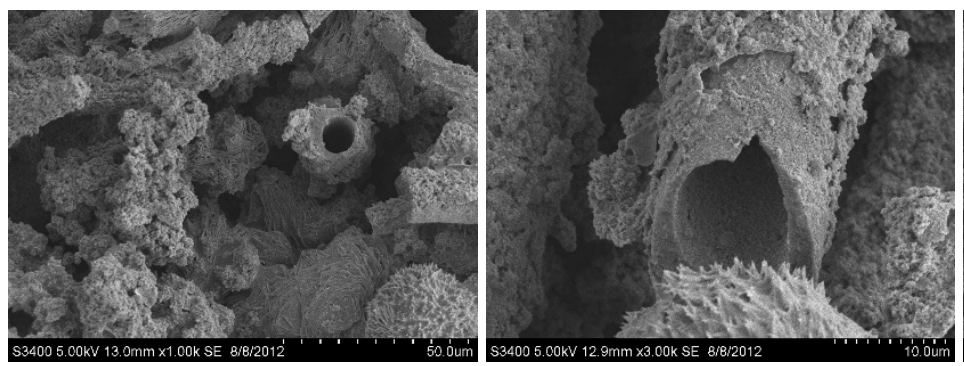

(a)
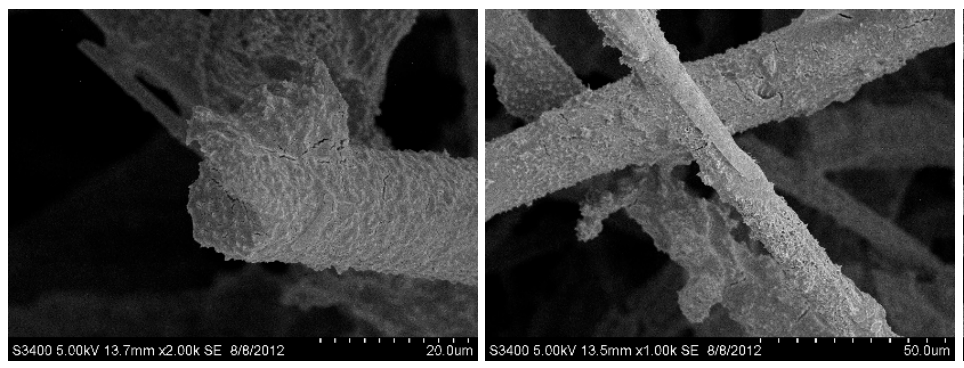

(b)
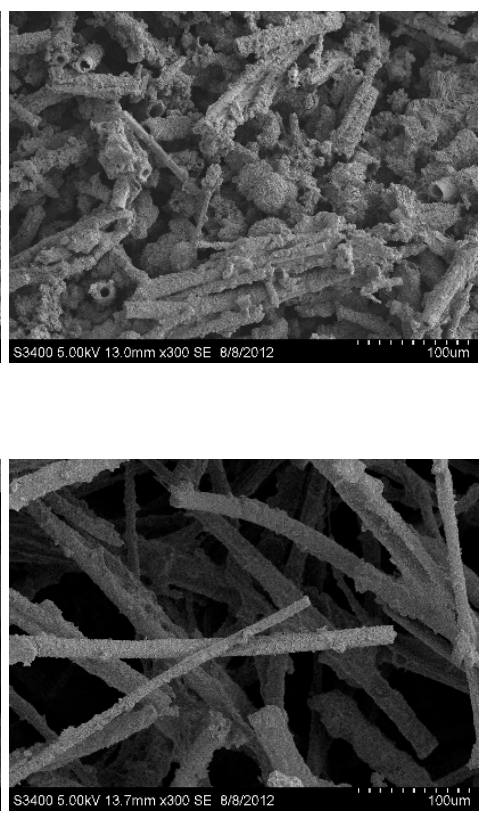

Fig.1. Contrast of scanning electron microscope photographs of ordinary fiber glass and those treated with silane coupling agent.

It can be seen from Figure 1(a) that after ordinary fiber glass is directly soaked in $\mathrm{NaOH}$ solution, the hollow structure appears in the fiber and serious powdering dregs occur on its surface; as for the fiber glass treated with the silane coupling agent in a way of infiltration, as shown in Figure 1(b), although the powdering appears on the surface, its powdering condition is significantly better that the untreated fiber glass. By contrast of scanning electron microscope photographs, it can be concluded that if silane coupling agent is coated on the surface of the fiber glass, it has alkali- resistance protective effect on the fiber glass.

\section{Effect of Silane Coupling Agent on Alkali-resistance Mass Loss Rate of the Fiber Glass.}

Fiber glass in a certain weight is soaked in $10 \% \mathrm{NaOH}$ solution for 1 hour and then rinse it with the distilled water, weigh it after drying and calculate fiber glass mass loss rate. Table 1 is contrast of the mass loss rate of ordinary fiber glass and modified fiber glass after soaking in $\mathrm{NaOH}$.

Table 2. The mass loss rate of modified fiber glass board after soaking in $\mathrm{NaOH}$

$$
\text { Ordinary fiber glass Modified fiber glass }
$$
Mass loss rate/\%
2.3
1.8

It can be seenfrom Table 2 that the silane coupling agent infiltration treatment can reduce alkali-resistance mass loss rate of the fiber glass to a certain extent, however the effect is not very obvious if its alkali-resistance treatment is only done by the coupling agent.

\section{Waterproof of Silane Coupling Agent}

Short-term water absorption volume can accurately and rapidly reflect the waterproof performance of the material. Adopt silane coupling agent in different concentrations to infiltrate fiber glass and 
evaluate its waterproof performance through the change of short-term water absorption volume. The short-term water absorption varying with KH550 concentration, as shown in Fig.2.

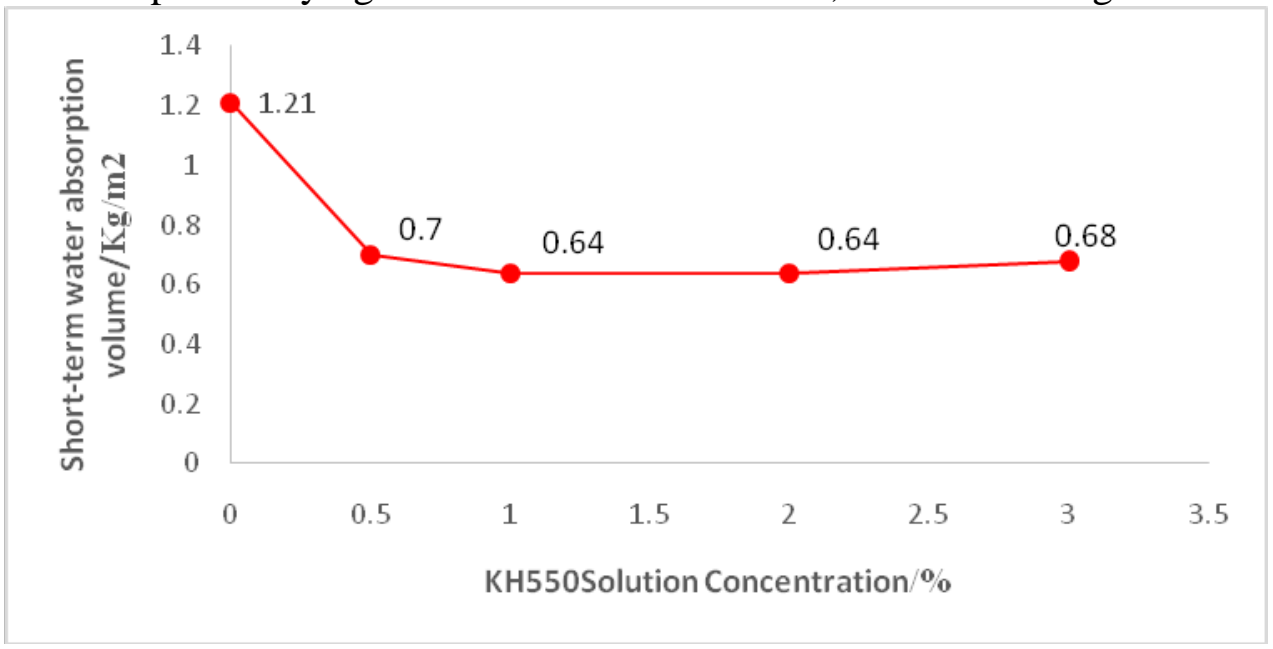

Fig.2. The short-term water absorption varying with KH550 concentration.

It can be seen from Figure 2 that the short-term water absorption volume of the fiber glass modified by silane coupling agent is significantly reduced in comparison with that of the ordinary glass fiber and its water-proof performance is enhanced by $50 \%-40 \%$. Silane coupling agent can effectively improve the waterproof performance of fiber glass. The short-term water absorption volume of the fiber glass is slightly reduced with the increase of silane coupling agent dosage but its effect is not obvious, even the water absorption volume increases when the treatment solution concentration is more than $3 \%$. Therefore, better water-proof effect can be obtained if it is not treated with silane coupling agent in high concentration.

\section{Comprehensive Performance Parameters of the Modified Fiber Glass Board}

Silane coupling agent infiltration treatment shows positive effect on water-proof and alkali resistance of fiber glass but it is to verify whether it will affect other properties of the fiber glass. In the experiment, adopt the contrast test method to investigate effect of silane coupling agent infiltration treatment on other property indicators (key indicators that may be affected by coupling agent). Test and analyze the key indicators of the modified fiber glass board by reference to combustion performance analysis of the national building materials and products (GB 8624-2012), monomer combustion test of the building materials or products(GB/T 20284-2006) and other standards and codes. The experimental results are shown in Table 3 below.

Fire proof performance of the modified fiber glass. Introduction of silane coupling agent may influence the fire rating of the fiber glass. However, it can be seen from Table 3 that the combustion heat value of fiber glass basically does not change when dosage of KH550 solution is controlled to be less than 3\%. Due to less dosage of KH550, it won't fireproof performance of the fiber glass. Therefore, the fiber glass treated with silane coupling agent can still maintain good fireproof performance and its combustion heat value can reach $2.58 \mathrm{MJ} / \mathrm{kg}$ and heat conduction coefficient up to $0.0328 \mathrm{~W} /(\mathrm{m} \bullet \mathrm{K})$.

Heat conduction coefficient of the modified fiber glass. It can be found from Table 3 that heat conduction performance of the fiber glass treated with silane coupling agent is basically same with that of the ordinary fiber glass.

Vertical pullout strength and compression strength of the modified fiber glass. It can be found from Table 3 that vertical pullout strength and compression strength of the infiltrated fiber glass basically show no change or are increased slightly. Because KH550 can increase binding force of fiber in the fiber glass, it shows binding effect but such force is little, additionally, due to little dosage of KH550, vertical pullout strength of the infiltrated fiber glass basically show no change or is 
increased slightly. Compression strength also has the same change rule, however, in comparison with the compression strength value, the coupling agent exercises less effect on the compression strength.

Table 3. Comprehensive performance parameters of the modified fiber glass board

\begin{tabular}{lcccc}
\hline & Untreated & $1 \%$ KH550 & 2\%KH550 & $3 \%$ KH550 \\
\hline $\begin{array}{l}\text { Calorific value of the } \\
\text { combustion }(\mathrm{MJ} / \mathrm{Kg})\end{array}$ & 2.58 & 2.57 & 2.58 & 2.58 \\
$\begin{array}{l}\text { Thermal conduction } \\
\text { coefficient }(\mathrm{W} /(\mathrm{m} \cdot \mathrm{K}))\end{array}$ & 0.0335 & 0.0331 & 0.0328 & 0.0342 \\
$\begin{array}{l}\text { Vertical pullout } \\
\text { strength (KPa) }\end{array}$ & 9.2 & 9.4 & 9.4 & 9.7 \\
$\begin{array}{l}\text { Compression strength } \\
(\mathrm{KPa})\end{array}$ & 41.2 & 40.9 & 42.2 & 41.8 \\
\hline
\end{tabular}

\section{Summary}

By investigating the water absorbing mechanism of glass fiber and its water proof performance and alkali erosion mechanism and alkali resistance, test fiber glass after the infiltration treatment of silane coupling agent. And investigate the effects on key performance indicators of fiber glass treated with silane coupling agent in a way of infiltration by the contrast test. The main conclusions are as follows.

(1)The infiltration treatment of silane coupling agent can improve waterproof per-formance and alkali resistance of glass fiber.

(2) The concentration of silane coupling agent shall be $0.5 \%-2 \%$. In order to maintain stability of silane coupling agent, $\mathrm{pH}$ value of silane coupling agent water solution shall be limited to 7-8 range.

(3)Silane coupling agent will not damage to comprehensive performance parameters of the modified fiber glass when its addition is less than $3 \%$ and it may slightly enhance the mechanical strength of fiber glass.

\section{References}

[1] Bo Zhou.Application of fiber glass and its products[J]. Plastic manufacturing, 2012, 05:74-75.

[2] Jiawei Yao, Zhaoming Hou, Yishu Yao. Fire resistance performance analysis of several thermal insulation material used in exterior wall of the building [J]. Low temperature architecture technology, 2012, 07:4-6.

[3] Yuan Yu.Performance analysis of thermal insulation materials used in exterior wall and application consideration [J]. China Building Materials Science and Technology, 2012, 04:52-55.

[4] Dunshi Ge. Kinetics investigation of glass fiber alkali erosion reaction of [J]. Glass fiber, 2005,05:4-9.

[5] WuYongkun, Fanghong Gong and Deren Sha. Study on alkali resistance of fiber glass [J]. Glass fiber, 2004, 04:6-14.

[6] Ping Hu, Ming Jiang, Chou Huang, Qian Yu. Study on the interface properties of silane coupling agent [J]. Surface technology, 2004, 05:19-21. 